\title{
A Novel Hybrid Threshold Proxy Signature Scheme
}

\author{
Xin Yang , Long Zhang* , Chunming Li , Guoqiang Li \\ School of Mathematical Sciences, Heilongjiang University, Harbin, 150080, China \\ E-mail: lzhang@hlju.edu.cn
}

\begin{abstract}
In this paper, we propose a new type of threshold proxy signature scheme: hybrid threshold proxy signature (HTPS), in which a signature is cooperatively generated by the partial original signers themselves and the proxy signers on behalf of their own original signer in threshold proxy way. We formalize the notion of security for hybrid threshold proxy signature scheme and prove our scheme's security, such as, distinguishability, verifiability, strong unforgeability, strong nonrepudiation, etc.
\end{abstract}

Keywords-threshold proxy signature; Hybrid threshold proxy signature scheme; ID-baesd

\section{INTRODUCTION}

In 1996, the concept of proxy signature was first introduced by Mambo et al ${ }^{[1]}$. It allows an original signer to delegate his signing power to a designated person, called the proxy signer, who has the power to act on behalf of the original signer. Following the development of proxy signature, the threshold proxy signature was also widely studied ${ }^{[2-4]}$. Threshold proxy signature schemes are designed to delegate the signing power to a proxy group of proxy signers. In a $(t, n)$ threshold proxy signature scheme, the proxy signature key is shared among a group of $n$ proxy signers delegated by the original signer. Any t or more proxy signers can cooperatively sign messages on behalf of the original signer.

Now, suppose there is a company with $n_{1}$ managers and $n_{2}$ secretaries. It is often needed that a document is signed by some department. If one or more managers needed in generating a signature are absent, according to Wang et al introduced hybrid proxy multisignature ${ }^{[5]}$, the secretary can take part in generating a multisignature on behalf of him.

But there is a disadvantage in Wang's scheme: if a manager and his proxy secretary are absent at the same time, Wang's scheme can't solve this problem.

To solve this problem, we introduce a new kind of threshold proxy signature: hybrid threshold proxy signature (HTPS). In a HTPS, the every absent manager delegate the signing power to a group of proxy signers for sharing signing responsibility, the group of proxy signers use $(t, n)$ threshold scheme on behalf of the absent manager, then proxy signers and the present managers cooperatively generate signature.

The HTPS is a special case of threshold proxy signature but more flexibility and functionality. The HTPS actually is

\footnotetext{
* Corresponding author. E-mail: lzhang@hlju.edu.cn
}

an arbitrary combination of the original signers' partial signatures and proxy signers' partial signatures. Especially, when all actual signers are original signers, the HTPS is an ordinary signature scheme; when all actual signers are proxy signers, the HTPS is an ordinary threshold proxy signature scheme.

In this paper, we formalize a notion of security for hybrid threshold proxy signature scheme. Then, we propose a new hybrid threshold proxy signature scheme and prove its security.

\section{NOTION OF HyBRID THRESHOLD PROXY SIGNATURE SCHEME}

\section{A. Definition of Hybrid Threshold Proxy Signature}

In a hybrid threshold proxy signature scheme, suppose there are $n_{1}$ departments and each department has an original signer and $n_{2}$ proxy signers as $\left(O_{i}, P_{i j}\right)$, here $P_{i j}$ is the $j$ proxy person in $O_{i}$ group $i=1, \cdots, n_{1}, j=1, \cdots, n_{2}$. There is a document needed to be signed by original signer jointly. If one or more original signers are absent, the proxy signers in the absent original signer group on behalf their respective original signer to generate proxy partial signature in $\left(t_{2}, n_{2}\right)$ threshold way. The final hybrid threshold proxy signature is cooperatively generated by the participant original signers and the proxy signers.

We call such a signature generated jointly by some ordinary signers and some proxy signers a hybrid threshold proxy signature.

\section{B. Syntax of Hybrid Threshold Proxy Signature Scheme}

The definition details the components of a hybrid threshold proxy signature scheme as following:

A hybrid threshold proxy signature scheme is a tuple $T P T S=\{G, S, V, P D C G, P S G, P S V, I D\}$

- $G$ The system parameters generation algorithm input the secure parameters $1^{\mathrm{k}}$, the algorithm output the public and private key for the original signers and the proxy signer are $\left(Q_{O_{i}}, S_{O_{i}}\right),\left(Q_{P_{i}}, Q_{P_{i}}\right)$.

- $S$ The standard signing algorithm, $S\left(m, S_{i}\right)=\sigma$, input: message $m \in\{0,1\}^{*}$ and secret key $S_{i}$, output : signature $\sigma$.

- $\quad V$ The verification algorithm, $V\left(m, \sigma, Q_{i}\right)=1$ or $\perp$, input message $m$, signature $\sigma$, public key of signer $Q_{i}$. If the signature is valid, output 1 , otherwise, output $\perp$. 
- $\quad$ PDCG The proxy delegation certificate generation algorithm, the original signers and the proxy signers generate $\operatorname{PDCG}\left(k_{O_{i}}, S_{O_{i}}, k_{P_{i j}}, S_{P_{i j}}\right)=\left(U_{O_{i} P}, S_{i}\right)$ cooperatively, input: random number and public key of each group, output: proxy delegation certificate of each group, then the clerk generate the final proxy delegation certificate $(U, S)$.

- HSG The hybrid signature generation algorithm, the participant original signers and the proxy signers in the absent original signers group collaboratively generate. $\operatorname{HSG}\left(m, m_{\omega},(U, S), S_{O_{i}}, S_{P_{i j}}\right)=\sigma$, input: message $m \in\{0,1\}^{*}$, secret key of actual signers $S_{O_{i}}, S_{P_{i j}}$, the proxy delegation certificate $q$ and a warrant $m_{\omega}$, and $m$ satisfies the requirements stated in $m_{\omega}$, output: hybrid proxy signature $h \sigma$.

- $\quad H S V$ The hybrid signature verification algorithm, 1.verify $(U, S)$. If it is hold, do the next, otherwise $\perp$; 2. $\quad H S V\left((U, S), m, m_{\omega}, h \sigma, Q_{O_{i}}, Q_{P_{i j}}\right)=0$ or 1 . Everyone can verify the valid of hybrid signature with this algorithm. If valid, output 1 , otherwise output 0 .

- ID The actual signers identification algorithm, input a hybrid threshold proxy signature $h \sigma$, output the actual signers identification, or $\perp$ in case of an error.

A hybrid threshold proxy signature is the extension of threshold proxy signature, so a security hybrid threshold proxy signature scheme also satisfies the security such as strong unforgeability, strong nonrepudiation, distinguishability, resistance to conspiracy attack, etc.

\section{A SECURE HyBRID THRESHOLD PROXY SigNATURE SCHEME}

\section{A. Setup}

PKG chooses $G_{1}$ and $G_{2}$ be two cyclic groups of prime $q$, Let $P$ be a generator of $G_{1}, e: G_{1} \times G_{1} \rightarrow G_{2}$ be a secure bilinear pairing, and choose two cryptographic hash function $H_{1}:\{0,1\}^{*} \rightarrow Z_{q}, H_{2}:\{0,1\}^{*} \rightarrow G_{1}$.PKG pick a random number $s$ from $Z_{q}^{*}$, compute $P_{p u b}=s P$, keep $s$ as secret key. Publish $\left\{G_{1}, G_{2}, e, q, P, P_{p u b}, H_{1}, H_{2}\right\}$.

\section{B. The Private Key Generation}

Given each original signer $O_{i}\left(i=1,2, \cdots, n_{1}\right)$ his identity $I D_{O_{i}}$, PKG computers $S_{O_{i}}=s Q_{O_{i}}=s H_{2}\left(I D_{O_{i}}\right)$ as private key.

Given each proxy signer $P_{i j}\left(i=1,2, \cdots, n_{1} ; j=1,2, \cdots, n_{2}\right)$ his identity $I D_{P_{i j}}$, PKG computers $S_{P_{i j}}=s Q_{P_{i j}}=s H_{2}\left(I D_{P_{i j}}\right)$ as private key.

\section{The Proxy Delegation Certificate Generation}

1) The original signers $O_{i},\left(i=1, \cdots, n_{1}\right)$ choose $k_{i}$ from $Z_{q}^{*}$, compute $U_{i}=k_{i} Q_{O_{i}}, h_{i}=H_{1}\left(m_{\omega} \| U_{i}\right), V_{i}=\left(k_{i}+h_{i}\right) S_{O_{i}}$. Here $m_{\omega}$ is proxy warrant which records the identities of the original signers and the proxy signers, correspondence, the parameters $t_{1}, t_{2}$, the valid delegation time, authorities. etc. $O_{i}\left(i=1, \cdots, n_{1}\right)$ chooses a random polynomial $f(x)$ over $G_{1}$ of degree $t_{2}-1$ :

$$
f_{i}(x)=V_{i}+a_{i 1} x+a_{i 2} x^{2}+\cdots+a_{i t_{2}-1} x^{t_{2}-1} .
$$

Computes $V_{P_{i j}}=f_{i}\left(I D_{P_{i j}}\right),\left(j=1,2, \cdots, n_{2}\right)$,

and sends $h_{i} V_{P_{i j}}$ to the proxy signers as their secret in secure ways, computes $A_{i j}=e\left(h_{i} V_{P_{i j}}, P\right), A_{i}=\prod_{j=1}^{n_{2}} A_{i j}$, and publish $\left(h_{i}, A_{i}, m_{\omega}, U_{i}\right)$.

2) Each proxy signer $P_{i j}$ verifies $e\left(h_{i} V_{P_{i j}}, P\right)=A_{i j}$ received $h_{i} V_{P_{i j}}$. If the verification is hold, accept, otherwise, reject.

3) $O_{i}\left(i=1,2, \cdots, n_{1}\right)$ chooses $k_{O_{i}}$ from $Z_{q}^{*}$, compute $U_{O_{i}}=e(P, P)^{k_{O_{i}}}$.

$P_{i j}\left(j=1,2, \cdots, n_{2}\right)$ chooses $k_{P_{i j}}$ from $Z_{q}^{*}$, compute $U_{P_{i j}}=e(P, P)^{k_{P_{i j}}}$, and publish $U_{O_{i}}, U_{P_{i j}}$.

Each group $O_{i}$ and $P_{i j}$ cooperatively compute

$$
U_{O_{i} P}=U_{O_{i}} \prod_{j=1}^{n_{2}} U_{P_{i j}}, h_{i}^{\prime}=H_{1}\left(m \| U_{O_{i} P}\right) .
$$

$O_{i}$ compute $s_{i}=S_{O_{i}} h_{i}^{\prime}+k_{O_{i}} P$

$P_{i j}$ compute $l_{i j}=S_{P_{i j}} h_{i}^{\prime}+k_{P_{i j}} P$, send $l_{i j}$ to $O_{i}$ in secure ways.

4) $O_{i}$ verifies $e\left(l_{i j}, P\right)=e\left(Q_{P_{i j}}, P_{p u b}\right)^{h_{i}^{\prime}} U_{P_{i j}}$ received $l_{i j}$.

If the verification is hold,

$O_{i}$ computes $S_{i}=s_{i}+\sum_{j=1}^{n_{2}} l_{i j}$, and sends $\left(U_{O_{i} P}, S_{i}\right)$ to $O_{1}$ and $P_{i j}$ as their proxy certificate.

5) After $O_{1}$ and $P_{i j}$ received $\left(U_{O_{i} P}, S_{i}\right)$, they verify

$$
e\left(S_{i}, P\right)=e\left(Q_{O_{i}}, P_{p u b}\right)^{h_{i}^{\prime}} e\left(\sum_{j=1}^{n_{2}} Q_{P_{i j}}, P_{p u b}\right)^{h_{i}^{\prime}} U_{O_{i} P} .
$$

If the verification is hold, accept $\left(U_{O_{i} P}, S_{i}\right)$.

$O_{1}$ compute $U=\prod_{i=1}^{n_{1}} U_{O_{i} P}, S=\sum_{i=1}^{n_{1}} S_{i}$ and publish $(U, S)$ as the final proxy delegation certificate.

\section{The Hybrid Threshold Proxy Signature Generation}

In order to sign message $m$, without loss of generality, we assume that $O_{1}, \cdots, O_{t_{1}}$ are the attendant original signers and $O_{t_{1}+1}, \cdots, O_{n_{1}}$ are the absent original signers, $P_{i 1}, \cdots, P_{i t_{2}}$ $\left(i=t_{1}+1, \cdots, n_{1}\right)$ are the actual proxy signers of absent original signers group.

1) $O_{i}\left(i=1, \cdots, t_{1}\right)$ open his $U_{O_{i}}$, every group’s actual 
proxy signers compute and publish

$$
U_{i}=\prod_{j=1}^{t_{2}} U_{P_{i j}}, i=t_{1}+1, \cdots, n_{1} .
$$

$O_{i}\left(i=1, \cdots, t_{1}\right), P_{i j}\left(i=t_{1}+1, \cdots n_{1} ; j=1, \cdots, t_{2}\right)$ cooperatively compute $D_{O P}=\prod_{i=1}^{t_{1}} U_{O_{i}} \prod_{i=t_{1}+1}^{n_{1}} U_{i}, v=H_{1}\left(m \| D_{O P}\right)$.

2) $P_{i j}\left(i=t_{1}+1, \cdots n_{1} ; j=1, \cdots, t_{2}\right)$ compute

$$
y_{P_{i j}}=\left(h_{i} V_{P_{i j}} \eta_{i j}+S_{P_{i j}}\right) v+k_{P_{i j}} P
$$

here $\eta_{i j}=\prod_{k=1, j \neq k}^{t_{2}}\left(-I D_{P_{i k}}\right)\left(I D_{P_{i j}}-I D_{P_{i k}}\right)^{-1}$,

every group verify $e\left(y_{P_{i j}}, P\right)=A_{i j}^{\eta_{i j} v} e\left(Q_{P_{i j}}, P_{p u b}\right)^{v} U_{P_{i j}}$,

if the verification is hold, compute $y_{P_{i}}=\sum_{j=1}^{t_{2}} y_{P_{i j}}$ as partial signature from $O_{i}$ to $P_{i j}$.

$O_{i}\left(i=1, \cdots, t_{1}\right)$ compute $y_{O_{i}}=S_{O_{i}} v+k_{O_{i}} P$ as his partial signature, publish $y_{O_{i}}, y_{P_{i}}$ to $O_{1}$ in secure ways.

3) $O_{1}$ received $y_{O_{i}}, y_{P_{i}}$, verifies

$$
\begin{gathered}
e\left(y_{O_{i}}, P\right)=e\left(Q_{O_{i}}, P_{p u b}\right)^{v} U_{O_{i}} \\
e\left(y_{P_{i}}, P\right)=e\left(h_{i}\left(U_{i}+h_{i} Q_{O_{i}}\right), P_{p u b}\right)^{v} e\left(\sum_{j=1}^{t_{2}} Q_{P_{i j}}, P_{p u b}\right)^{v} U_{i}
\end{gathered}
$$

If the verification is hold, computes

$$
Y=\sum_{i=1}^{t_{1}} y_{O_{i}}+\sum_{i=t_{1}+1}^{n_{1}} y_{P_{i}} .
$$

The proxy signature on $m$ is $\left(m, m_{\omega},(U, S),(v, Y)\right)$.

\section{E. Proxy Signature Verification}

To verify a proxy signature $\left(m, m_{\omega},(U, S),(v, Y)\right)$ for message $m$, any verifier performs the following steps:

1) Verifies proxy certificate $(U, S)$ with verification $e(S, P)=e\left(\sum_{i=1}^{n_{1}} Q_{O_{i}}, P_{p u b}\right)^{h_{i}^{\prime}} e\left(\sum_{i=1}^{n_{1}} \sum_{j=1}^{n_{2}} Q_{P_{i j}}, P_{p u b}\right)^{h_{i}^{\prime}} U$

2)

$$
\begin{aligned}
D_{O P}= & e(Y, P)\left[e\left(h_{i} \sum_{i=t_{1}+1}^{n_{1}}\left(U_{i}+h_{i} Q_{O_{i}}\right), P_{p u b}\right)\right. \\
& \left.e\left(\sum_{i=1}^{t_{1}} Q_{O_{i}}, P_{p u b}\right) e\left(\sum_{i=t_{1}+1}^{n_{1}} \sum_{j=1}^{t_{2}} Q_{P_{i j}}, P_{p u b}\right)\right]^{-v}
\end{aligned}
$$

if and only if $v=H_{1}\left(m \| D_{O P}\right)$ accept this proxy signature.

\section{CORRECTNESS AND SECURITY ANALYSIS}

\section{A. Correctness Analysis}

1) The proxy delegation certificate is valid because of the following:

$$
\begin{aligned}
& e(S, P)=e\left(\sum_{i=1}^{n_{1}} S_{i}, P\right)=e\left(\sum_{i=1}^{n_{1}}\left(S_{i}+\sum_{j=1}^{n_{2}} l_{i j}\right), P\right) \\
&=e\left(\sum_{i=1}^{n_{1}}\left(S_{O_{i}} h_{i}^{\prime}+k_{O_{i}} P\right)+\sum_{i=1}^{n_{1}} \sum_{j=1}^{n_{2}}\left(S_{P_{i j}} h_{i}^{\prime}+k_{P_{i j}} P\right), P\right) \\
&=e\left(\sum_{i=1}^{n_{1}} S_{O_{i}} h_{i}^{\prime}, P\right) e\left(\sum_{i=1}^{n_{1}} k_{O_{i}} P, P\right) e\left(\sum_{i=1}^{n_{1}} \sum_{j=1}^{n_{2}} S_{P_{i j}} h_{i}^{\prime}, P\right) \\
& e\left(\sum_{i=1}^{n_{1}} \sum_{j=1}^{n_{2}} k_{P_{i j}} P, P\right) \\
&= e\left(\sum_{i=1}^{n_{1}} Q_{O_{i}}, P_{p u b}\right)^{h_{i}^{\prime}} e\left(\sum_{i=1}^{n_{1}} \sum_{j=1}^{n_{2}} Q_{P_{i j}}, P_{p u b}\right)^{h_{i}^{\prime}} U
\end{aligned}
$$

2) The verifier can verify $v=H_{1}\left(m \| D_{O P}\right)$ to prove hybrid threshold proxy signature $\left(m, m_{\omega},\left(U_{P}, S\right),(v, Y)\right)$ on $m$.

$e(Y, P)=e\left(\sum_{i=1}^{t_{1}} y_{O_{i}}+\sum_{i=t_{1}+1}^{n_{1}} y_{P_{i}}, P\right)$

$=e\left(\sum_{i=1}^{t_{1}}\left[S_{O_{i}} v+k_{O_{i}} P\right]+\sum_{i=t_{1}+1}^{n_{1}} \sum_{j=1}^{t_{2}} y_{P_{i j}}, P\right)$

$=e\left(\sum_{i=1}^{t_{1}} S_{O_{i}} \nu, P\right) e\left(\sum_{i=1}^{t_{1}} k_{O_{i}} P, P\right) e\left(\sum_{i=t_{1}+1}^{n_{1}} \sum_{j=1}^{t_{2}} h_{i} V_{P_{i j}} \eta_{i j} \nu, P\right)$

$e\left(\sum_{i=t_{1}+1}^{n_{1}} \sum_{j=1}^{t_{2}} S_{P_{i j}} \nu, P\right) e\left(\sum_{i=t_{1}+1}^{n_{1}} \sum_{j=1}^{t_{2}} k_{P_{i j}} P, P\right)$

$=e\left(h_{i} \sum_{i=t_{1}+1}^{n_{1}}\left(U_{i}+h_{i} Q_{O_{i}}\right), P_{p u b}\right)^{v} e\left(\sum_{i=1}^{t_{1}} Q_{O_{i}}, P_{p u b}\right)^{v}$

$e\left(\sum_{i=t_{1}+1}^{n_{1}} \sum_{j=1}^{t_{2}} Q_{P_{i j}}, P_{p u b}\right)^{v} D_{O P}$

$D_{O P}=e(Y, P)\left[e\left(h_{i} \sum_{i=t_{1}+1}^{n_{1}}\left(U_{i}+h_{i} Q_{O_{i}}\right), P_{p u b}\right) e\left(\sum_{i=1}^{t_{1}} Q_{O_{i}}, P_{p u b}\right)\right.$

$\left.e\left(\sum_{i=t_{1}+1}^{n_{1}} \sum_{j=1}^{t_{2}} Q_{P_{i}}, P_{p u b}\right)\right]^{-v}$

So $v=H_{1}\left(m \| D_{O P}\right)$

\section{B. Security Analysis}

Like the general threshold proxy signature, the hybrid threshold proxy signature scheme should satisfy the following requirement:

1) Distinguishability

The valid proxy signature contains warrant $m_{\omega}$, there are the public key of original signers and proxy signers in verification equation.

2) Verifiability

In a valid proxy signature $\left(m, m_{\omega},(U, S),(v, Y)\right)$ on $m$, the verifier can be convinced of the original signer delegated the proxy signers, because there is proxy delegation certificate $(U, S)$ in proxy signature.

3) Strong unforgeability

Firstly, in proxy signature verification, the verifier verifies proxy certificate $(U, S)$ which contains secret, private keys of original signers and private keys of proxy signers, the others unknown these parameters. Secondly, the signers use Hess scheme to generate proxy signature, Hess scheme ${ }^{[6]}$ has already verified Security in the insider-attack and outsider-attack.

4) Strong nonrepudiation

Verification equation contains original signers and proxy signers' public keys, once a proxy signer creates a valid proxy signature of an original signer, he can't repudiate the signature creation.

5) Resistance to conspiracy attack

The clerk send blind factors instead of $V_{P_{i j}}=f_{i}\left(I D_{P_{i j}}\right)$, to the users, so anyone can't get the secret polynomial $f(x)$.

6) Prevention of misuse

The valid proxy signature contains warrant $m_{\omega}$ and $m_{\omega}$ contains the valid delegation time authorities, etc.

\section{CONCLUSIONS}

In this paper, we propose a new threshold proxy signature: hybrid threshold proxy signature scheme. Firstly, 
we formalize a notion of security for hybrid threshold proxy signature scheme, and then we propose an identity-based hybrid threshold proxy signature scheme with the bilinear pairing. With analyzing, we find our scheme has many secure characters, such as, distinguishability, verifiability, strong unforgeability, strong nonrepudiation, resistance to conspiracy attack, prevention of misuse, etc.

\section{ACKNOWLEDGMENT}

The authors thank the editors and the anonymous referees for their valuable comments and suggestions. This work is supported by Scientific Research Fund of Heilongjiang Provincial Education Department (Grant No. 12521405).

\section{REFERENCES}

[1] Mambo M, Usuda K, and Okamoto E. Proxy signature: Delegation of the Power to sign messages. IEICE Trans. on Fundatamentals of
Electronics, Communications and Computer Sciences 1996, E79-A(9): 1338-1354.

[2] Zhang K. Threshold proxy signature schemes. In: Proceedings of the Information Security Workshop (ISW'97), Lecture Notes in Computer Science, Springer-Verlag, 1998, 1396: 282-290.

[3] S Kim, S Park, and D Won. Proxy signatures, revisited. In: Proceedings of the Information and Communications Security ( ICICS'97), Lecture Notes in Computer Science, Springer-Verlag, 1997, 1334:223-232.

[4] Sun H M, Lee N Y, and Hwang T.Threshold proxy signatures.IEE Proceedings-Computers and Digital Techniques, 1999, 146(5): 259263.

[5] Zecheng Wang, Haifeng Qian,and Zhibin Li. Hybrid proxy multisignature: A new type multi-party signature. Information Sciences, 2007, 177(6): 5638-5650.

[6] Hess F.Efficient identity based signature schemes based on pairings. Proceedings of Selected Areas in Cryptography 2002, Newfoundland, Canada, 2002, 310-324. 